\title{
Quality of Service in Wireless Sensor Networks
}

\author{
Mohammed Q. AL_shamiry \\ Faculty of Computer and \\ Information Technology \\ Sana'a University, Sana'a, Yemen
}

\author{
Sharaf A. Alhomdy, PhD \\ Faculty of Computer and \\ Information Technology \\ Sana'a University, Yemen
}

\author{
Yosef A. Abdulmoghni \\ Faculty of Computer and Information \\ Technology \\ Al- Razi University, Sana'a, Yemen
}

\begin{abstract}
Wireless Sensor Network (WSN) is a widespread network used in many daily life activities such as environment, health, and nuclear radiation. Since, WSNs are networks that consists of sensors which are distributed in an ad hoc manner. Therefore, there are many important issues related to WSN such as energy consumption, end-to-end delay, and packets delivery ratio. This study aims to review the most recent works published in the field of quality of service (QoS) metrics in WSNs. It focuses on energy consumption, end-to- end delay and packets delivery ratio metrics. It will be helpful for researchers to know some of the trends and recent research in field of networking, as well as to learn some future works in this area.
\end{abstract}

\section{General Terms}

Power consumption, end-to-end delay, network lifetime, routing protocols.

\section{Keywords}

Wireless Sensor Networks (WSNs), Base Station (BS), Quality of Service (QoS), Internet of Things (IoT).

\section{INTRODUCTION}

Wireless Sensor Networks (WSNs) are networks that consists of sensors which are distributed in an ad hoc manner. These sensors work with each other to sense some physical phenomenon and then gathering the information to get relevant results. But they have low power, limited memory, and energy constrained due to their small size [1]. The sensor node lifetime typically exhibits a strong dependency on battery life. In many cases, the wireless sensor node has a limited power source $(<500 \mathrm{mAh}, 1.2 \mathrm{~V})$, and replenishment of power may be limited or impossible altogether [2][3].

The roles of WSN engineers is to develop a cost-effective standards-based wireless networking solution that supports lowto medium data rates, has low power consumption, and guarantees security and reliability. The position of sensor nodes do not have be predetermined, allowing random deployment in inaccessible terrains or dynamic situations; however, this also means that sensor network protocols and algorithms must possess self-organizing capabilities. For military and/or national security applications, sensor devices must be amenable to rapid deployment. The deployment must be supportable in an ad hoc fashion, and the environment is expected to be highly dynamic [4].

Traditionally, sensor networks have been used in the context of high-end applications such as radiation and nuclear-threat detection systems, "over-the-horizon" weapon sensors for ships, biomedical applications, habitat sensing, and seismic monitoring. More recently, interest has focusing on networked biological and chemical sensors for national security applications; furthermore, evolving interest extends to direct consumer applications. Existing and potential applications of sensor networks include, among others, military sensing, physical security, air traffic control, traffic surveillance, video surveillance, industrial and manufacturing automation, process control, inventory management, distributed robotics, weather sensing, environment monitoring, national border monitoring, and building and structures monitoring [5][6]. There are different types of sensors as Seismic, thermal, visual, and infrared sensors.

WSN has two basic kinds of architecture: Layered Architecture, and Clustered Architecture. A layered architecture has a single powerful base station (BS), and the layers of sensor nodes around it correspond to the nodes that have the same hop-count to the BS. The advantage of a layered architecture is that each node is involved only in short-distance, low-power transmissions to nodes of the neighboring layers. A clustered architecture organizes the sensor nodes into clusters, each governed by a cluster-head. The nodes in each cluster are involved in message exchanges with their cluster-heads, and these heads send message to a BS. Clustered architecture is useful for sensor networks because of its inherent suitability for data fusion. The data gathered by all member of the cluster can be fused at the clusterhead, and only the resulting information needs to be communicated to the BS. The cluster formation and election of cluster-heads must be an autonomous, distributed process [7][8].

Operational Challenges of WSNs are energy efficiency, limited storage and computation, low bandwidth and high error rates, scalability to a large number of sensor nodes, survivability in harsh environments, experiments are time- and space-intensive, and Errors are common as wireless communication, noisy measurements, and node failure are expected [1]. There three common objectives from a Quality of Service (QoS) perspective in WSN, those are energy efficiency, reliable communication and latency awareness [1]. There are many obstacles in the development of WSNs. Therefore, many universities and technology companies employ groups of researchers to overcome these obstacles. This study focused on review some QoS metrics in WSN.

\section{OVERVIEW}

There are many of literature review that focused on QoS metrics in WSN. This study gathers 29 scientific papers. These papers classified into three categories. The first category is the Energy Consumption in WSN. The second category is the end-to-end delay. The third category is packets delivery ratio to Base Station (BS).

\subsection{Energy Consumption}

The WSN consists of a group of small sensors that have a limited power source. Energy consumption is one of the most important issues related to the lifetime of WSN. Many researchers have focused on energy consumption, and how to reduce energy consumption.

In 2018, Youngbok Cho an et al. proposed hierarchical network architecture to solve the energy consumption problem, and implement suitable routing mechanism to handle low power devices (devices battery operated ). The simulation results showed that the proposed scheme is more energy efficient and 
flexible than traditional wireless sensor network schemes and consequently, the new scheme can be implemented for efficient communication in the Internet of Things. The proposed approaches didn't focus on end-to-end delay and packets delivery ratio [4].

EiP-LEACH is protocol proposed by Anupkumar M Bongale et al. [9] aims at enhance energy consumption in WSN, in which using probability based threshold value that is influenced by residual energy of candidate Cluster Header node and average initial energy of entire network for Cluster Header selection, their proposed reduces energy consumption when the distance is small between the Cluster Header and the Base Station, in their protocol only energy is considered the primary parameter in deciding Cluster Header node. But they did not care about the distance to Base Station.

Ons Bouachir et al.[10] presented an EAMP-AIDC protocol, an energy aware MAC protocol for EH-WSNs based on individual duty cycle optimization. It takes into consideration nodes' residual energy and data and application requirements to define active and sleep periods (individual dynamic duty cycles), that allow to create a balanced load in term of energy consumption and in terms of cooperative data relaying tasks between the different participating nodes so as to ensure continuous network operation. OMNET++ was used to evaluate the proposed protocol, the simulation results showed that the proposed protocol is better in energy consumption compared to the IEEE 802.15.4 standard. However, EAMP-AIDC MAC and data dissemination/aggregation protocols aren't related, and it didn't focus on packets delivery ratio and end-to-end delay.

In [11], Mohammad Fouladlou et al. proposed a routing algorithm in improve the energy efficiency of WSN devices in Internet of Things. The propose used the genetic algorithm for clustering to reduce energy consumption in wireless nodes. The compared their algorithm with IEEE 802.15.4 protocol. The proposed routing algorithm has shown a better performance in terms of energy consumption. The proposed algorithm doesn't focus on other QoS metrics in WSN, such as end-to-end delay and Loss in WSN.

Wided Abidi, and Tahar Ezzedine.[12] proposed a new algorithm for clustering in WSN ,they use fuzzy logic. Their, proposed approach is based on three parameters, distance between the node and the Base Station, residual energy , and the number of neighbors within the cluster range to elect Clusters Header and form clusters. Their simulation results confirm that their approach is effective to reduce energy and increase the lifetime of the WSN. But, they didn't focus on end-to-end delay and packets delivery ratio in WSN.

In [13], Walid Abushiba et al. proposed $\mathrm{CH}-$ leach for improves energy consumption to WSN in IOT environment. Simulation results show that their proposed protocol improves network life by $40 \%$ compared to DEEC protocol. The authors in this approach didn't focus on other QoS metrics in WSN, such as of LCP according to other networking metrics, such as packets delivery ratio and end-to-end delay.

In [8], Rana Helal, and Amr ElMougy. proposed a new protocol for local Service Discovery (SD), that propose eliminates the need for a dedicated gateway. The proposed protocol utilizes a multi-tier network architecture in order to achieve two objectives: high success rate in satisfying service requests, and energy efficiency. By limiting the number of hops that an Service Discovery request has to traverse before being satisfied, Energy efficiency is achieved, and by using a hierarchical structure of Distributed Hash Tables (DHTs) a high success rate is end-to-end delay and Loss in WSN.

EHARA is a new routing algorithm proposed by Thien D. Nguyen et al.[14] they integrating a new parameter called Extra Backoff for enhanced the algorithm. Simulation results showed that their algorithm enhanced network lifetime by about $50 \%$ Compared to Path Recovery Time algorithm, It also enhanced the network lifetime by $40 \%$ compared to Energy Harvesting Aware Ad-hoc On-Demand Distance Vector Routing Protocol (EHA-AODV) algorithm. The algorithm did not focus on other networking metrics, such as packets delivery ratio and end-to-end delay in WSN.

ICH-LEACH is protocol proposed by M. Bennani Mohamed Taj et al.[15] it uses at most one intermediate $\mathrm{CH}$. The aim of their protocol is to extend the lifetime of the WSN. The proposed protocol is characterized by the adjustment of the power of transmission, and the distance between the BS and cluster heads. The proposed protocol increases the life time of the Wireless Sensor Network, and reduces energy consumption. The authors did not care about packets delivery ratio in the Wireless Sensor Network.

In [16], Jian Shen et al. proposed a new Energy Efficient Centroid-based Routing Protocol (EECRP) for wireless sensor network assisted internet of things to improve the performance of the network and increase the network lifetime. The proposed protocol includes three key parts: a new series of algorithms for adapting clusters and rotating $\mathrm{CH}$ for evenly distribute the energy load among all nodes in the network, a new distributed cluster formation technique, and a new mechanism to reduce the energy consumption. The proposed protocol includes three key parts: a new series of algorithms for adapting clusters and rotating $\mathrm{CH}$ for evenly distribute the energy load among all nodes in the network, a new distributed cluster formation technique, and a new mechanism to reduce the energy consumption. Their simulation results showed that EECRP performs better than GEEC, and LEACH-C to reduce energy consumption. However, EECRP isn't suitable when the BS is located outside of the network, and it isn't focus on other networking metrics, such as end-to-end delay and packets delivery ratio in WSN.

Mohamed Eshaftri et al. [7] proposed Load-balancing ClusterBased Protocol (LCP), that is a new Cluster Based protocol that introduces a new inter-cluster approach to increase WSN lifetime. The proposed protocol rotates continuously the election of the $\mathrm{CH}$ election in each cluster according to highest residual energy in each round. The main contribution of their proposed protocol is its ability to rotate the role of the Cluster Header between nodes of the same group, and selecting the node with the highest energy to become a Cluster Header. The simulation results showed that the LCP protocol increases network lifetime by $15 \%$ compared to other clustering protocols. However, energy consumption were the performance metrics used in the evaluation of the LCP, but they didn't evaluate the performance

guaranteed .The simulations results showed that the proposed SD protocol achieves energy efficiency without sacrificing success rate of serving requests. The proposed approach didn't implement a hardware prototype of this protocol in order to evaluate its robustness on a real network, also, the authors didn't focus on delay.

LEACH-TLCH is algorithm proposed by Chunyao FU et al.[17]. it is a new improved algorithm of LEACH protocol which is intended to extend the life of the network and balance the energy consumption of the entire network. The simulation results indicated that there is an improvement in energy efficiency and 
lifetime of the network. The authors didn't focus on other networking metrics, such as packets delivery ratio and end-toend delay.

\subsection{End-to-End Delay}

Delay in the transmission of data between the sensors with each other or between the sensors and the BS is an important problem in WSN. Many researchers have focused on end-to-end delay and how to reducing end-to-end delay. Next sections show reviews on eight scientific research papers related to End-to-End Delay issues.

In [18], Yan Sun et al. proposed Speed Up-Greedy Perimeter Stateless Routing Protocol for WSNs (SU-GPSR), it is a position-based routing scheme. The greedy mode works together with speed up mode to provide a solution for data transmission. SU-GPSR not only embraces both mobile nodes and still nodes in one unified scheme, but when evaluating the potential next hop, SU-GPSR provide a complete energy consumption consideration. The simulation results showed that the SU-GPSR has a improvement on average transmission delay compared with EGPSR and FA-GPSR. The proposed protocol did not care about the packets delivery rate.

Sabin Bhandari et al.[19] proposed a new cloud-assisted clustering framework, where each sensor node in their proposed framework elects itself as a $\mathrm{CH}$ on the basis of the closeness factor, and residual energy. They analyzed the impact of heterogeneity of Internet of Things networks in terms of bondness among IoT nodes and energy. The numerical results show that the proposed framework reduces End to End delay, and provides higher throughput and longer network stability as compared to the conventional one. The proposed framework is not suitable for Internet of Vehicle (IoV) network, the proposed framework don't prioritization for wireless IoT.

In [20] Mengkun et al. proposed a new dynamic network routing protocol for WSN, through improved the GPSR protocol based on stratification of traffic density. The main idea of improved the GPSR protocol is using a stratification strategy, depending on the density of nodes nearby, Where the nodes can adjust strategy of maintaining neighbors list. Nodes maintain a two- hopsneighbor list, when the density of nodes nearby is lower than critical value. Nodes maintain a one-hop neighbor list, when the density of nodes nearby is larger than critical value. Simulation experimental results showed their the performance of improved algorithm has a improvement on average transmission delay compared with the classical GPSR algorithm, and also the transmission overhead was smaller than the 2- hop GPSR protocol. The authors did not focus on Energy consumption in WSN.

two energy-efficient models proposed by Ademola et al.[6] for WSNs in the Internet of Things environment, the first model is an Energy-Aware Clustering model, and the second model is an a Service-Aware Clustering model. In the first model individual sensor nodes are assigned roles based on their service delivery. Simulation results showed that their proposed models reduces data packets delay and has the longest network lifetime compared with LEACH-C and FCMCP protocols. The proposed approach did not compare a service-aware clustering model with multiple BSs .

In [21], Alka Singh et al. discussed selection cluster head and vice cluster head and presented a method of energy efficient routing based on both V-LEACH protocol and particle swarm optimization (PSO) technique. The proposed protocol reduces End to End delay, reduces energy consumption, and increases the life time of the WSN. But they didn't focus on packets delivery ratio in WSN.

Enhanced GPSR (E-GPSR) is protocol proposed by Tianli Hu et al.[22] aims at reduce the end-to-end delay in WSN, the proposed protocol into account takes the length of nodes' residual buffer and the geographical location of the nodes. The simulation results show that this proposed protocol reduces the end-to-end delay. E-GPSR did not focus on Energy consumption.

In [23], Demir AK et al. proposed cross-layer approach using the network and MAC layer to improve the delay, reliability and throughput in WMSNs. In this approach, each node has to know the quality level of the next hop to select the best route to the sink. The quality level here is a function of channel quality, data rate, and buffer size and accordingly the next hop is chosen.

Lijuan Wang et al.[24] proposed a new routing protocol called GPSRI (Greedy Perimeter Stateless Routing Improved), which an improvement of a GPSR. The simulation results showed that the GPSRI Reduces the end-to-end delay rate in WSN. GPSRI didn't focus on other QoS metrics in WSN, such as energy consumption and packets delivery ratio.

\subsection{Packets Delivery Ratio}

There are many threats and factors affecting the successful access of data to BS. Data loss is an important problem in WSN. Many researchers have focused on Packets Delivery Ratio, and how to increase the packets delivery rate to BS. Next sections show reviews on nine scientific research papers related to packets delivery ratio issues.

Hongyan xin and Xuxun liu.[25] proposed an accurate-distances based transmission scheme, to solve the many-to-one transmission problem of strip-based Wireless Sensor Network and avoid energy holes of such a Wireless Sensor Network, which is divided into multiple layers. This scheme didn't care about transmission design assisted by super nodes, and they didn't care in delay in WSN.

In [26], Hui Chen et al. proposed an improved inter-cluster multi-hop routing protocol based on quality of service (CMPBQ), that base on traditional LEACH protocol, this protocol aims to ensure reliable data transmission in WSNs. The simulation results showed that the proposed protocol improve the data transmission reliability. In this approach, the authors didn't focus on delay and Energy consumption in WSN.

Hasna Dhehibi et al.[27] proposed new design of energy efficient LEACH protocol that based on MOD LEACH. They tried to manage intelligently the remain power of the node in order to increase lifetime of the network and packets delivery ratio. Their results demonstrated that their proposed protocol well improve lifetime of the network and packets delivery ratio to the base station. This model did not focus on end-to-end delay.

GRCS is new geographic routing protocol proposed by Manel Khelifi et al.[28] that enhance of the GPSR protocol, the proposed protocol based on the clustering mechanism for WSNs. This protocol aims to increase packets delivery ratio successfully to the BS. They in their proposed protocol had optimized the path to the destination dynamically by selecting some different paths to reach the destination. Simulation results confirmed that GRCS, when compared to GPSR, presents better rate of packets delivery successfully to the BS. However, in GRCS protocol, the locations of the destination and the source are used to route the packets. and the routing decision is not always based on routing tables. In such a type of routing, imprecise location information 
can have devastating consequences on the routing process

In [29], Varun Sharma and Somesh Sharma. improved the performance of health monitoring network by LEACH protocol (Low Energy Adaptive Clustering Hierarchy) Protocol. They reduced the energy consumption and packet loss ratio for the mobile health monitoring so that Patient Health Record (PHR) can transfer securely. The simulation results showed the Packets Loss Ratio and Energy Consumption Be better. The authors did not focus on delay in WSN.

M. Faheem et al.[30] proposed a new dynamic clustering protocol, it based quality-of-service (QoS) and energy efficient (called EQRP). The proposed protocol reduces excessive packets retransmissions and improves network reliability significantly for WSN-based the Smart Grid (SG) applications

Performance results show that the EQRP has successfully improved packets delivery ratio, improved throughput ,residual energy, and memory utilization. The proposed protocol for WSN-based the Smart Grid (SG) applications only and not for all WSN-based IOT applications.

In[31], Emad Alnawafa et al. proposed an improved multi-hope technique (IMHT-LEACH). The IMHT-LEACH distributes all the Cluster Heads into a number of levels. It suggested a new technique to route the data to the BS through the levels. The simulation results indicated that the proposed protocol improves the lifetime, throughput, stability of the WSN, and increase rate of packets delivery to the BS. The proposed approach didn't focus on the delay in WSN.

Chinmaya Mahapatra et al.[32] proposed a Data Aware Energy Efficient Distributed Clustering Internet (DAEECI) protocol by saving Cluster Header selection energy using active Radio Frequency Identification (RFID) tags. The proposed protocol aims at increase the rate of successful delivery of data with less energy. They formulated Energy Consumption models in each round data is sent from sensor nodes to Base Station through gateway nodes. The results showed that the Internet Of Things based Wireless Sensor Network heterogeneous systems are better equipped to deal with data delivery and energy efficiency problems, and increase rate of packets delivery to the BS. Simulation results supported their proposed method and showed significant improvement over state of the art techniques. The authors did not focus on delay in WSN.

Energy-Efficient LEACH (EE-LEACH) is Protocol for data gathering introduced by Gopi Saminathan et al.[33] it in order to reduce power consumption for routing in WSN based on optimal clustering and the effective data ensemble. In this system, a cluster head is elected for each clusters to optimize the resource utilization. The Energy-Efficient routing can be obtained by nodes which have the maximum residual energy. Hence, the highest residual energy nodes are selected to forward the data to Base Station. The proposed protocol can improve the network lifetime. EE-LEACH did not care about protect the network from security attacks, and the delay in WSN.

\section{DISCUSSIONS}

QoS in WSN is a hot research topic. Delay, loss, lifetime, and energy consumption are the most metrics the researchers concentrate on. WSN components dedicated to work isolated from each other without permanent power resource. This make many challenges and trade off problems if aiming at satisfying the QoS metrics. Therefore, researchers often concentrate on satisfying a specific metric according to the requirements of the application the network is dedicated for. Some researchers focused on reducing energy consumption, while others focused on reducing end-to-end delay, and other researchers focused on packets delivery rate. Some researchers have focused on improving two QoS metrics, such as reducing power consumption and reducing end-to-end delay, or reducing power consumption and increasing packets delivery rate. As shown before, there are a few studies focused on improving all QoS metrics in WSN such as power consumption, end-to-end delay, and packets delivery.as mention before, considering all the metrics may cause a tradeoff problem between the other metrics.

\section{CONCLUSIONS}

This review paper shows that there is considerable interest in developing the WSN in terms of energy consumption, end-toend delay, and packets delivery. There are many studies in this field have a significant contribution in improving the QoS in the WSN. This study focused on some scientific papers that concentrate on QoS in WSN. This study focused on energy consumption, end-to-end delay, and packets delivery ratio in WSN. Despite the development of the WSN, there is a need for further improvement on these three QoS metrics. in WSN. The previous studies focused on one metric or two metrics of QoS metrics and did not focus on the other metrics. So, including all the other metrics will be considered as a future work.

\section{REFERENCES}

[1] Lina Xu, Rem Collier, and Gregory M. P. O'Hare, Member, IEEE, (2017), "A Survey of Clustering Techniques in WSNs and Consideration of the Challenges of Applying Such to 5G IoT Scenarios" JOURNAL OF IEEE INTERNET OF THINGS, VOL. 6, NO. 1.

[2] Kawaljeet Kaur and Kiranbir Kaur, (2016)," A Study of Power Management Techniques for Internet of Things (IoT)" International Conference on Electrical, Electronics, and Optimization Techniques (ICEEOT).

[3] E. Shih et al., (2001), "Physical Layer Driven Protocol and Algorithm Design for Energy- Efficient Wireless Sensor Networks," Proceedings of the 7th ACM International Conference on Mobile Computing and Networking (MobiCom'01), Rome, Italy, pp. 272-286.

[4] W. Su et al.,(2004), "Communication Protocols for Sensor Networks," in Wireless Sensor Networks, C. S. Raghavendra, K. Sivalingam, and T. Znati, Eds., Kluwer Academic, New York.

[5] Youngbok Cho, Minkang Kim, and Sunghee Woo,(2018), " Energy Efficient IoT based on Wireless Sensor Networks for Healthcare " International Conference on Advanced Communications Technology(ICACT).

[6] Ademola . Abidoye, et al,(2017), "Models for integrating wireless sensor networks into the Internet of Things", IET Wirel. Sens. Syst, Vol. 7 Iss. 3, pp. 65-72.

[7] Mohamed Eshaftri, Ahmed Yassin Al-Dubai, et al, ( SEPTEMBER 2015), "A New Energy Efficient Cluster based Protocol for Wireless Sensor Networks" CONFERENCE PAPER, http://www.researchgate.net/publication/278672754.

[8] Rana Helal, Amr ElMougy,(2015)," An Energy-Efficient Service Discovery Protocol for the IoT based on a MultiTier WSN Architecture" 40th Annual IEEE Conference on Local Computer Networks.

[9] Anupkumar M Bongale, Anand Swarup et al,(2017), " EiPLEACH: Energy influenced Probability based LEACH Protocol for Wireless Sensor Network", International Conference on Emerging Trends \& Innovation in ICT 
(ICEI).

[10] Ons Bouachir, Adel Ben Mnaouer et al,(2017), "EAMPAIDC - Energy-Aware Mac Protocol With Adaptive Individual Duty Cycle For EH-WSN", IEEE , 978-1-50904372-9/17.

[11] Mohammad Fouladlou, and Ahmad Khademzadeh,(2017)," An Energy Efficient Clustering Algorithm for Wireless Sensor Devices in Internet of Things", Artificial Intelligence and Robotics (IRANOPEN).

[12] Wided Abidi , and Tahar Ezzedine,(2017), " Fuzzy Cluster Head Election Algorithm based on LEACH protocol for Wireless Sensor Networks" , IEEE , 978-1-5090-43729/17.

[13] Walid Abushiba, Princy Johnson, et al, (2017), "An Energy Efficient and Adaptive Clustering for Wireless Sensor Network (CH-leach) using Leach Protocol " IEEE, 978-1$5386-4266-5 / 17$

[14] Thien D. Nguyen, Jamil Y. Khan, et al,(2017)," An Effective Energy-Harvesting-Aware Routing Algorithm for WSN-based IoT Applications", IEEE ICC Green Communications Systems and Networks Symposium.

[15] M. Bennani Mohamed Taj, and M. AIT KBIR,(2016), "ICH-LEACH: An enhanced LEACH protocol for Wireless Sensor Network", IEEE , 978-1-5090-6227-0/16.

[16] Jian Shen, Member, et al, (AUGUST 2015),"An Efficient Centroid-based Routing Protocol for Energy Management in WSN Assisted IoT" JOURNAL OF LATEX CLASS FILES IEEE, VOL. 14, NO. 8.

[17] Chunyao FU , Zhifang JIANG, et al,( January 2013), " An Energy Balanced Algorithm of LEACH Protocol in WSN", IJCSI International Journal of Computer Science Issues, Vol. 10, Issue 1, No 1.

[18] Yan Sun, Junpeng Guo, et al,( July 2017), "Speed UpGreedy Perimeter Stateless Routing Protocol for Wireless Sensor Networks (SU-GPSR)", IEEE .

[19] Sabin Bhandari, Shree Krishna Sharma, et al,(2017), " Cloud-Assisted Device Clustering for Lifetime Prolongation in Wireless IoT Networks" , IEEE 30th Canadian Conference on Electrical and Computer Engineering (CCECE).

[20] Mengkun , Huan , et al,( July 2017)," An Improved GPSR Protocol Based on Stratification of Traffic Density", International Conference on Information and Automation (ICIA) Macau SAR, China.

[21] Alka Singh, Shubhangi Rathkanthiwar , et al,( April 6-8, 2016), "Energy Efficient Routing of WSN using Particle Swarm Optimization and V-Leach Protocol",International Conference on Communication and Signal Processing,
India.

[22] Tianli Hu,Minghui Liwang, Lianfen Huang, Yuliang Tang, (July 22-24, 2015),"An Enhanced GPSR Routing Protocol based on the buffer length of nodes for the Congestion problem in VANETs", The 10th International Conference on Computer Science \& Education (ICCSE 2015).

[23] Demir AK, Demiray HE, et al,(2014): cross-layer QoS architecture for wireless multimedia sensor networks. Wireless networks; 20:655-670.

[24] Lijuan Wang, and Haitao Liang,(2012)," Research and improvement of the wireless sensor network routing algorithm GPSR", International Conference on Computing, Measurement, Control and Sensor Network.

[25] Hongyan Xin and Xuxun Liu,( September 2017)," EnergyBalanced Transmission With Accurate Distances for StripBased Wireless Sensor Networks", IEEE.

[26] Hui Chen, Feng Wang et al,(2017), "An Improved InterCluster Multi-hop LEACH Protocol Oriented Reliable Transmission in WSNs" 3rd IEEE International Conference on Computer and Communications.

[27] Hasna Dhehibi, Ahmed Zouinkhi, et al, (2017)," An Enhancement of MOD-LEACH protocol for Wireless Sensor Network ", International Multi-Conference on Systems, Signals \& Devices (SSD).

[28] Manel Khelifi, Slimane Bourouais, et al,(2017)," GRCS: A Cluster-based Geographic Routing Protocol for WSNs" ICUFN.

[29] Varun Sharma, and Somesh Sharma,(2017), " Low Energy Consumption based Patient Health Monitoring by LEACH Protocol" International Conference on Inventive Systems and Control (ICISC)

[30] M. Faheem, and V.C. Gungor,(2017), "Energy efficient and QoS-aware routing protocol for wireless sensornetworkbased smart grid applications in the context of industry 4.0", ELSEVIER

[31] Emad Alnawafa, and Ion Marghescu,(2017), "IMHT: Improved MHT-LEACH Protocol for Wireless Sensor Networks",8th International Conference on Information and Communication Systems (ICICS).

[32] Chinmaya Mahapatra , Zhengguo Sheng, et al,(2016), Energy-efficient and Distributed Data-aware Clustering Protocol for the Internet-of-Things " IEEE Canadian Conference on Electrical and Computer Engineering (CCECE).

[33] Gopi Saminathan, Arumugam, et al,(2015), "EE-LEACH: development of energy-efficient LEACH Protocol for data gathering in WSN",Arumugam and Ponnuchamy EURASIP Journal on Wireless Communications and Networking. 